\title{
BMJ Open Efficacy of adding a physiotherapy rehabilitation programme to arthroscopic management of femoroacetabular impingement syndrome: a randomised controlled trial (FAIR)
}

Kim L Bennell, ${ }^{1}$ Libby Spiers, ${ }^{1}$ Amir Takla, ${ }^{2}$ John O’Donnell, ${ }^{3}$ Jessica Kasza, ${ }^{4}$ David J Hunter, ${ }^{5,6}$ Rana S Hinman ${ }^{1}$

To cite: Bennell KL, Spiers L, Takla A, et al. Efficacy of adding a physiotherapy rehabilitation programme to arthroscopic management of femoroacetabular impingement syndrome: a randomised controlled trial (FAIR). BMJ Open 2017;7:e014658. doi:10.1136/ bmjopen-2016-014658

- Prepublication history and additional material are available. To view these files please visit the journal online (http://dx.doi.org/ 10.1136/ bmjopen-2016-014658).

Received 10 0ctober 2016 Revised 20 March 2017 Accepted 31 March 2017

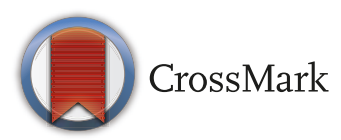

For numbered affiliations see end of article.

Correspondence to Dr Kim L Bennell; k.bennell@ unimelb.edu.au

\section{ABSTRACT}

Objectives Although several rehabilitation programmes following hip arthroscopy for femoracetabular impingement (FAl) syndrome have been described, there are no clinical trials evaluating whether formal physiotherapy-prescribed rehabilitation improves recovery compared with self-directed rehabilitation. The objective of this study was to evaluate the efficacy of adding a physiotherapist-prescribed rehabilitation programme to arthroscopic surgery for FAl syndrome. Design Randomised controlled trial.

Methods People aged $\geq 16$ years with FAl syndrome scheduled for hip arthroscopy were recruited and randomly allocated to physiotherapy (PT) or control. The PT group received seven PT sessions (one preoperative and six postoperative) incorporating education, manual therapy and a progressive rehabilitation programme of home, aquatic and gym exercises while the control group did not undertake PT rehabilitation. Measurements were taken at baseline ( 2 weeks presurgery) and 14 and 24 weeks postsurgery. The primary outcomes were the International Hip Outcome Tool (iHOT-33) and the sport subscale of the Hip Outcome Score (HOS) at week 14.

Results Due to slower than expected recruitment and funding constraints, recruitment was ceased after 23 months. Thirty participants (14 PT and 16 control) were randomised and 28 (14 PT and 14 control; 93\%) and 22 (11 PT and 11 control; 73\%) completed week 14 and 24 measurements, respectively. For the 14-week primary outcomes, the PT group showed significantly greater improvements on the iHOT-33 (mean difference 14.2 units; $95 \% \mathrm{Cl} 1.2$ to 27.2 ) and sport subscale of the HOS (13.8 units; $95 \% \mathrm{Cl} 0.3$ to 27.3). There were no significant between-group differences at week 24.

Conclusions An individual PT treatment and rehabilitation programme may augment improvements in patientreported outcomes following arthroscopy for FAl syndrome. However, given the small sample size, larger trials are needed to validate the findings.

Trial registration number Trial registered with the Australian New Zealand Clinical Trials Registry :ACTRN12613000282785, Results.

\section{Strengths and limitations of this study}

- Our study was designed with attention to key methodological features to minimise bias including randomisation, concealed allocation and intentionto-treat analysis.

- The primary outcomes were reliable and valid patient-reported measures suitable for young active individuals with femoracetabular impingement syndrome.

- The delivery of the physiotherapy intervention by multiple physiotherapists enhances the generalisability of the findings.

- The results cannot necessarily be generalised to other physiotherapy protocols with different content and number of physiotherapy sessions.

- The trial recruited fewer than expected participants and hence given the small sample size, the results should be considered preliminary and require replication in a larger study.

\section{INTRODUCTION}

Femoroacetabular impingement (FAI) syndrome is a common cause of hip/groin symptoms and impaired performance in younger sporting populations ${ }^{1-3}$ and a potential precursor to hip osteoarthritis. ${ }^{4-6}$ FAI syndrome results from morphological hip abnormalities where the proximal femur and non-bony connective tissue abut against the acetabular rim. ${ }^{2}$

Hip arthroscopy is often used to manage FAI syndrome ${ }^{7}$ with case series generally reporting favourable outcomes. ${ }^{89}$ Use of postoperative rehabilitation is variable and dependent on surgeon's preferences and patient access to services. ${ }^{10}$ Furthermore, content of rehabilitation programmes vary considerably. ${ }^{10}$ 
Although several rehabilitation programmes following hip arthroscopy have been described, ${ }^{10-13}$ there are no clinical trials evaluating whether formal physiotherapy (PT)-prescribed rehabilitation improves recovery compared with self-directed rehabilitation.

Thus, the objective of this clinical trial was to evaluate the efficacy of a progressive physiotherapist-prescribed rehabilitation programme in individuals undergoing hip arthroscopy for FAI syndrome. The primary hypothesis was that those receiving physiotherapist-prescribed rehabilitation would report significantly greater improvements in health-related quality-of-life and function in sport at 14 weeks postsurgery than those in the control group not undergoing PT rehabilitation.

\section{METHODS}

We conducted a parallel-design two-arm randomised controlled trial (RCT) with outcomes assessed at baseline (within 2 weeks prior to surgery), at 14 weeks postsurgery (immediately following the PT intervention) and at 24 weeks postsurgery (figure 1). Ethical approval was obtained from the institutional Human Research Ethics Committee (\#1238190). Participants provided written informed consent. The trial protocol has been published. ${ }^{12}$ Part way through recruitment, the original upper age limit of 35 years was removed, and patients undergoing bilateral surgery were included to improve generalisability and expedite recruitment. The funding organisation did not have a role in the collection, analysis or interpretation of data, or in approval of publication of this manuscript.

Individuals aged $\geq 16$ years with FAI syndrome (diagnosed by an orthopaedic surgeon based on clinical/ imaging findings) with hip/groin symptoms for $\geq 3$ months and scheduled for hip arthroscopy were recruited from the private surgical practices of three orthopaedic surgeons in Melbourne, Australia. Exclusion criteria included: (1) radiographic evidence of hip osteoarthritis more than mild in severity defined as Tönnis $>\operatorname{grade} 1^{14}$; (2) professional athlete; (3) other concurrent injury/ condition affecting ability to undertake rehabilitation; (4) unable to attend a study physiotherapist if randomised to the PT group; (5) unwilling to refrain from formalised PT rehabilitation; and (6) unable to understand English.

Potentially eligible patients were identified by study surgeons and provided with study information. An independent research assistant confirmed eligibility via subsequent telephone screening. Consenting participants completed baseline questionnaires electronically approximately 2 weeks before surgery. Participants were then consecutively randomised into either the physiotherapist-prescribed rehabilitation (PT) group or the control group.

A study biostatistician prepared the randomisation schedule (computer-generated random permuted blocks of $6-8$, stratified by orthopaedic surgeon and unilateral/bilateral surgery). Consecutively numbered, sealed, opaque envelopes containing group allocation were prepared by an independent researcher. Envelopes were stored in a locked location and opened in sequence to reveal group allocation by a different researcher not involved in recruitment or data handling.

It was not possible to blind participants (who were also deemed to be assessors given that outcome measures were self-reported) or physiotherapists providing the intervention. However, surgeons performing hip arthroscopy, the physiotherapists providing inpatient management and the biostatistician were blinded to group allocation.

All participants underwent hip arthroscopic surgery for FAI syndrome performed by an experienced hip orthopaedic surgeon. Unstable articular cartilage flaps were debrided and exposed subchondral bone was treated by microfracture if $<400 \mathrm{sq} \mathrm{mm}$. Superficial labral tears were debrided, and tears that were unstable or $>50 \%$ deep were repaired. Partial or complete teres tears were debrided with a radiofrequency probe. Cam impingement lesions were treated by femoral osteochondroplasty. Pincer impingement lesions were treated when both radiological and intraoperative pathological evidence confirmed the diagnosis.

Immediate postoperative care was consistent for both groups and included: ice and compression; one inpatient visit by the hospitals' physiotherapist for provision of a gait aid; surgeons' usual written educational material; and use of a non-steroidal anti-inflammatory drug. A follow-up visit with the surgeon occurred approximately 2 weeks postsurgery. Patients were advised to use crutches until pain-free walking, likely 5 days or less, and to avoid hip flexion past $90^{\circ}$ and positions that could cause impingement and/or increase inflammation for approximately 6 weeks.

The protocol for the physiotherapist-prescribed rehabilitation has been previously described. ${ }^{12}$ It was a progressive semistructured accelerated programme based on the Takla-O'Donnell protocol, developed and refined by two of the authors over 10 years and currently used in their clinical practice (see table 1, online supplementary material 1 , online supplementary material 2) ${ }^{12}$ Participants in the PT group attended seven $30 \mathrm{~min}$ individual appointments with a study physiotherapist: one preoperative visit (after baseline assessment) within 2 weeks prior to surgery and six postoperative visits commencing at week two (approximately 2, 4, 6, 8, 10 and 12 weeks postsurgery). Six physiotherapists with $\geq 2$ years of musculoskeletal experience were trained to provide the intervention.

The PT programme included both mandatory and optional components and comprised of standardised PT assessments/reassessments, education and advice, manual therapy techniques, daily home exercise programme, unsupervised gym and aquatic programme at a local community facility at least twice weekly and graduated return to sport and physical activity. Individual progression of the programme was guided by the assessment findings and the surgical intervention, such 


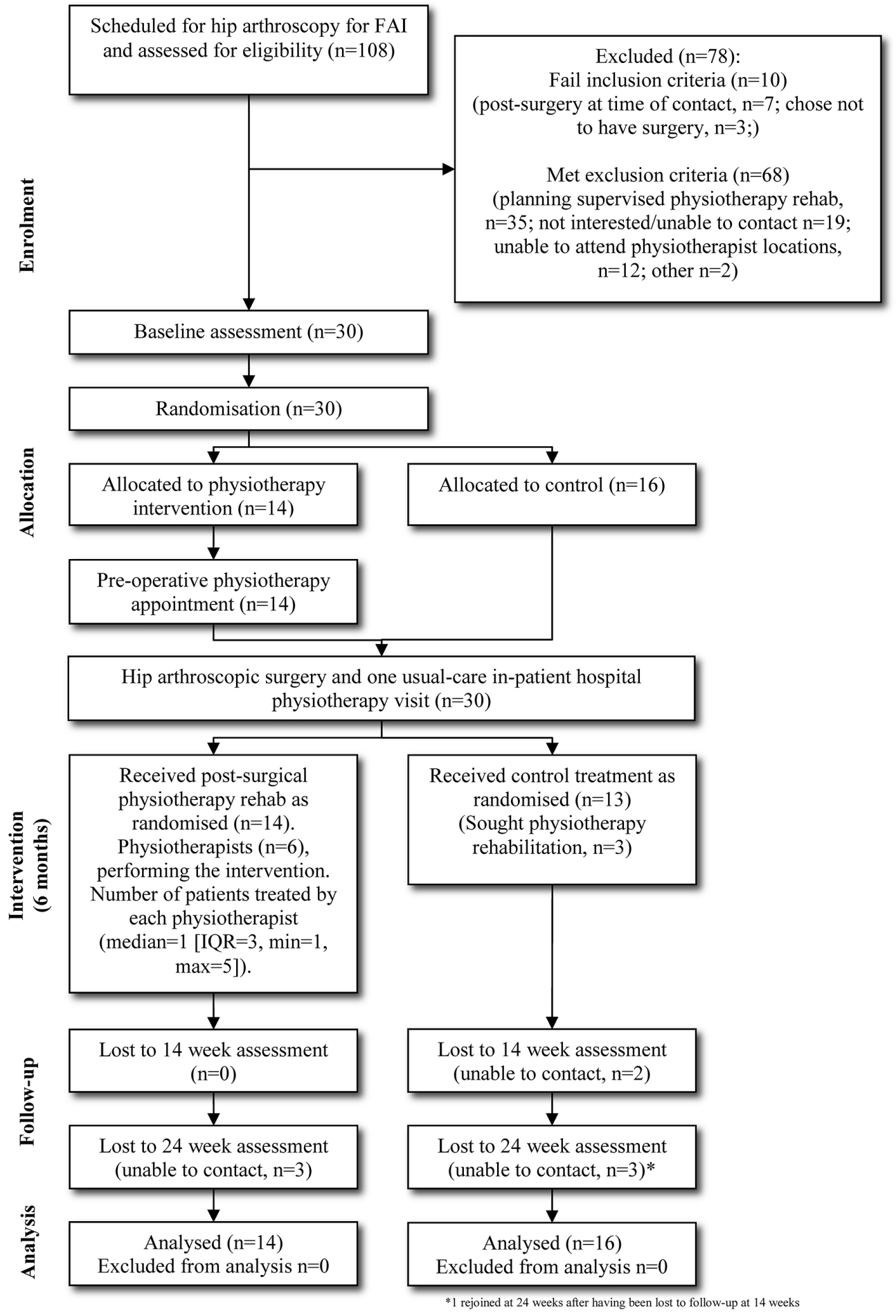

Figure 1 Participant flow through the trial. FAl, femoracetabular impingement.

as whether microfracture was used. PT treatment and gym/pool access were provided free of charge to the PT group participants.

Education: education and advice was the key component of the preoperative treatment session and an important part all postoperative session. Participants were given a handout summarising information about postoperative joint protection, including activities to avoid or modify. Advice was given about return to driving and work, and the importance of the home exercise programme was emphasised.

Manual therapy: trigger point massage was used at each postsurgical treatment session to release muscle tension, assist with pain relief and improve hip range of motion. Lumbar spine mobilisation (passive accessory intervertebral movements) was used when the PT assessment indicated it was needed. 


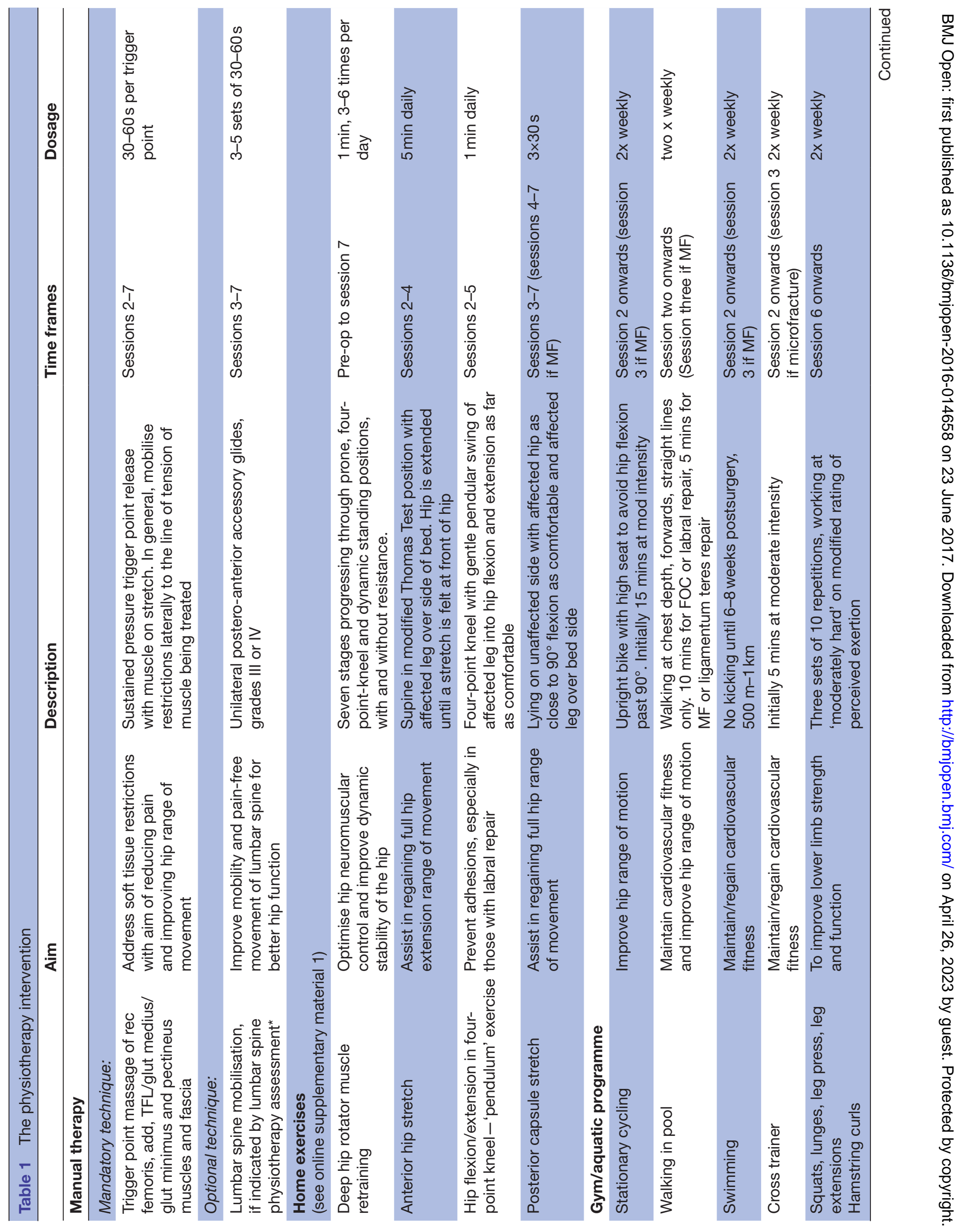


Home exercise programme: the daily home exercise programme aimed to improve local stabilisation of the hip joint by retraining and strengthening the deep hip rotator muscles. This muscle retraining followed seven stages, with participants moving to the next stage once they achieved effective activation and endurance of the muscles as determined by the therapist. Exercises to reduce the risk of adhesions and maximise hip range of movement were also prescribed. Exercise sheets were provided to study participants (see online supplementary material 1).

Gym and aquatic programme: participants were provided with access to local community gym and pool facilities (YMCA centres) and asked to attend at least twice weekly following the first postoperative PT session. Participants walked in the pool and used a stationary bike and cross-trainer, progressing to swimming and lower body resistance exercise.

Return to sport: this included provision of functional and sport-specific drills. Preliminary components of sporting activity generally commenced $6-8$ weeks postsurgery, with training in the actual sporting environment starting 10-12 weeks postsurgery.

The control group received no physiotherapist-prescribed rehabilitation programme. Participants were asked to refrain from seeing a physiotherapist for the first 3 months after surgery. Participants gradually increased their physical activity levels and returned to exercise/ sport based on information provided by the surgeon. As such, participants performed self-directed rehabilitation.

Physiotherapist protocol adherence was maximised through provision of a treatment manual, attendance at a 1-day training course and telephone meetings. Physiotherapists used standardised treatment recording forms, which were audited by research staff.

Age, sex, occupation, sporting involvement, duration of hip symptoms, previous treatments, medication use, surgical findings and surgical intervention were obtained from questionnaires and the surgical report.

The International Hip Outcome Tool (iHOT-33) measures health-related quality-of-life in young active patients with hip disorders ${ }^{15}$ and covers: symptoms and functional limitations (16 items); sports and recreational activities (six items); job-related concerns (four items); and social, emotional and lifestyle concerns (seven items). It uses a $100 \mathrm{~mm}$ horizontal visual analogue scale where a higher score represents better quality-of-life. This tool has good test-retest reliability, demonstrated face, content and construct validity and is highly responsive to clinical change. ${ }^{1516}$ The minimum clinically important difference (MCID) for this patient population is 6.1. ${ }^{15}$

The Hip Outcome Scale (HOS) assesses the degree of difficulty in performing tasks in: activities of daily living (ADLs; 17 items) and sport (nine items). ${ }^{17}$ Items are scored on a 5-point Likert scale with scores summed and converted to a percentage. In younger patients undergoing hip arthroscopy, the HOS has excellent test-retest reliability, ${ }^{16}$ evidence of content, construct 
and concurrent validity ${ }^{19}$ and is responsive to clinical change. ${ }^{20}$ The MCID for the sport subscale is 6.0. ${ }^{20}$

The Copenhagen Hip and Groin Outcome Score (HAGOS) assesses hip and groin disability, ${ }^{21}$ including pain (10 items), symptoms (7 items), physical function in daily living (5 items), physical function in sport and recreation (8 items), participation in physical activities (2 items) and hip and/or groin-related quality-of-life (5 items). Items are scored on a 5-point Likert scale and a normalised score (100 indicating no symptoms and 0 indicating extreme symptoms) is calculated. In young to middle-aged patients with chronic hip/groin pain, the HAGOS has good to excellent test-retest reliability ${ }^{1621}$ and evidence of construct validity and responsiveness to change. ${ }^{21}$

At each reassessment, participants rated their perceived overall change in their hip/groin problem (compared with baseline) on a seven-point ordinal scale (much worse to much better). ${ }^{22}$

A modified Tegner Activity Scale ${ }^{23}$ (scores 0-10 with 10 indicating professional/international level competition) and the Heidelberg Sports Activity Score $(\text { HSAS })^{24}(0-220$, higher score indicating greater sports activity) were administered to grade participants' level of sport/recreation and occupational physical activity.

The number of PT visits attended was recorded for the PT group. The PT group maintained a log-book to record home exercise, gym and pool activity. The number of exercise sessions completed was calculated as a percentage of those prescribed by the physiotherapist. In addition, participants self-rated their adherence to the rehabilitation programme at 14 weeks postsurgery on an 11-point numeric rating scale ( 0 being 'not at all' to 10 being 'completely as instructed').

Information on adverse events, cointerventions and medication use was collected from all participants during the intervention phase using a log-book and by self-report questionnaire at 14 and 24 weeks postsurgery.

Primary endpoints were change from baseline to 14weeks postsurgery in: (1) the iHOT-33 and (2) sports subscale of the HOS. Between-participant SDs have not been widely reported in the literature for these questionnaires so the study was powered to detect a moderate effect size of 0.5. Given this, the required sample for a two-tailed comparison of the groups using analysis of covariance with baseline values as covariates and an assumed correlation between participant measures at baseline and 14 weeks of $0.6^{25}$ when $\mathrm{d}=0.5$, power is 0.8 and type $\mathrm{I}$ error is .05 was 41 participants per group.

Analysis was by a blinded biostatistician (JK) using Stata (V.13.1) software on an intention-to-treat basis. Testing was two sided with a significance level set at $\mathrm{p}<0.05$. Due to minimal missing data (2 out of 30 participants for week 14 outcomes), a complete case analysis was performed rather than analysis using multiple imputation. Betweengroup differences in mean change from baseline to each time point were compared using linear regression modelling adjusting for baseline levels of the outcome measure and including physiotherapist as a random effect to account for clustering. Ratings of global change were dichotomised a priori as improved ('moderately' or 'much' better) and not improved ('slightly' better and below) and between-group comparisons made using log binomial regression and presented as relative risks.

\section{RESULTS}

Participants were recruited between July 2013 and June 2015. Figure 1 shows participant flow through the trial. Due to difficulty recruiting participants, we terminated recruitment prior to the target number. Of 108 patients screened, $78(72 \%)$ were ineligible or did not wish to participate. In total, 30 participants (14 PT, 16 control) were randomised and 28 (14 PT, 14 control; 93\%) and 22 (11 PT, 11 control; 73\%) completed week 14 and 24 measurements, respectively. Cross-over occurred from control to PT, with three (19\%) participants seeking PT rehabilitation prior to week 14. Characteristics of treatment groups and intraoperative findings and surgical procedures (table 2) were similar between groups, except for slightly more acetabular rim lesions and microfractures in the PT group.

At week 24, there were no significant differences between groups for changes in any primary or secondary outcome (table 3). A similar proportion of participants in both groups reported overall improvement (PT: 9/11, $82 \%$ vs controls $8 / 10,80 \%$; relative risk $1.0 ; 95 \%$ CI 0.67 to 1.6$)$.

All participants in the PT group attended all seven PT appointments. The mean (SD) percentage of prescribed exercise sessions completed was excellent at $88.8(10.0) \%$. On a scale of $0-10$, mean (SD) self-rated adherence was 8.3 (1.2) for the overall programme. For individual programme components, self-rated adherence was 8.6 (1.4) for home exercises, 5.9 (3.5) for the pool programme and 7.2 (2.4) for the gym programme.

Two participants in the PT group reported mild adverse events comprising muscle soreness from exercise and back pain. Use of other cointerventions was minimal and included analgesia (PT: $n=2$, control: $n=2$ ). The three participants who crossed over from the control group sought massage in addition to PT, while another two consulted a health professional in the first 14 weeks after surgery (osteopathy and yoga, $\mathrm{n}=1$; chiropractic, $\mathrm{n}=1$ ).

\section{DISCUSSION}

One of the major advantages of hip arthroscopic surgery for FAI syndrome is the relative speed of recovery compared with open surgery. ${ }^{26}$ Any additional intervention that further speeds recovery would add to this perceived advantage. We found that a physiotherapist-prescribed rehabilitation programme led to greater improvements of a clinically relevant magnitude for both primary outcomes at 14 weeks postsurgery compared with a patient-managed protocol with only minor input from an inhospital physiotherapist and the surgeon. At 
Table 2 Baseline characteristics and intraoperative findings and surgical management by group, reported as mean (SD), unless otherwise stated

\begin{tabular}{lcc}
\hline & $\begin{array}{l}\text { Physiotherapy } \\
(\mathbf{n = 1 4 )}\end{array}$ & $\begin{array}{l}\text { Control } \\
(\mathbf{n}=16)\end{array}$ \\
\hline Age (years) & $31.0(7.0)$ & $28.6(8.1)$ \\
\hline Male, $\mathrm{n}(\%)$ & $12(86)$ & $12(75)$ \\
\hline Symptom duration (years) & $5.0(4.6)$ & $3.3(2.6)$ \\
\hline Height (m) & $1.81(0.07)$ & $1.75(0.09)$ \\
\hline Body mass (kg) & $82.8(12.4)$ & $75.8(11.6)$ \\
\hline Body mass index (kg/m²) & $24.6(2.2)$ & $25.2(3.2)$ \\
\hline Unilateral surgery, $\mathrm{n}(\%)$ & $13(93)$ & $14(88)$ \\
\hline Dominant leg $\mathrm{n}(\%)$ & $6(43)$ & $8(50)$ \\
\hline Currently employed, $\mathrm{n}(\%)$ & $10(71)$ & $13(81)$ \\
\hline Previous treatment usage, $\mathrm{n}(\%)$ & $16(100)$ \\
\hline Stretching & $10(71)$ & $14(88)$ \\
\hline Strengthening & $11(79)$ & $13(81)$ \\
\hline $\begin{array}{l}\text { Oral anti-inflammatory } \\
\text { medication }\end{array}$ & $11(79)$ & $12(75)$ \\
\hline Physiotherapy & $11(79)$ & $10(63)$ \\
\hline Massage & &
\end{tabular}

Highest level of sporting

competition, $\mathrm{n}(\%)$

\begin{tabular}{|c|c|c|}
\hline $\begin{array}{l}\text { Elite/subelite (international/ } \\
\text { national) }\end{array}$ & $5(36)$ & $6(38)$ \\
\hline State & $7(50)$ & $3(19)$ \\
\hline Recreational & $2(13)$ & $5(31)$ \\
\hline Never competed & 0 & $2(13)$ \\
\hline \multicolumn{3}{|l|}{$\begin{array}{l}\text { Current level of sporting } \\
\text { competition, } n(\%)\end{array}$} \\
\hline $\begin{array}{l}\text { Elite/subelite (international/ } \\
\text { national) }\end{array}$ & 0 & $1(6)$ \\
\hline State & 0 & $1(6)$ \\
\hline Recreational & $4(29)$ & $8(50)$ \\
\hline Not competing & $10(71)$ & $6(38)$ \\
\hline \multicolumn{3}{|l|}{ Intraoperative findings, n (\%) } \\
\hline Cam FAl & $8(57)$ & $9(56)$ \\
\hline Pincer FAI & $1(7)$ & $2(13)$ \\
\hline $\begin{array}{l}\text { Combined FAl (cam and } \\
\text { pincer) }\end{array}$ & $5(36)$ & $5(31)$ \\
\hline $\begin{array}{l}\text { Ligamentum teres } \\
\text { pathology }\end{array}$ & $9(64)$ & $12(75)$ \\
\hline Labral disorder & $7(50 \%)$ & $7(44 \%)$ \\
\hline Synovitis & $3(21 \%)$ & $5(31 \%)$ \\
\hline Acetabular rim lesion & $13(93 \%)$ & $11(69 \%)$ \\
\hline \multicolumn{3}{|l|}{ Surgical management, n (\%) } \\
\hline Femoral ostectomy & $13(93)$ & $12(80)$ \\
\hline Acetabular ostectomy & $6(43)$ & $7(44)$ \\
\hline Labral repair & $6(43)$ & $4(25)$ \\
\hline
\end{tabular}

Table 2 Continued

\begin{tabular}{lcc} 
& $\begin{array}{l}\text { Physiotherapy } \\
(\mathbf{n}=14)\end{array}$ & $\begin{array}{l}\text { Control } \\
(\mathbf{n}=16)\end{array}$ \\
\hline $\begin{array}{l}\text { Ligamentum teres } \\
\text { debridement }\end{array}$ & $9(64)$ & $12(75)$ \\
$\begin{array}{l}\text { Capsular shrinkage } \\
\text { Microfracture }\end{array}$ & $0(0)$ & $1(6)$ \\
\hline
\end{tabular}

Continuous outcomes across time points are summarised in table 3 , and changes within- and between-groups in table 4. For the 14-week primary outcomes, the PT group showed significantly greater improvements compared with controls on the iHOT-33 (mean difference 14.2 units; $95 \% \mathrm{Cl} 1.2$ to $27.2, \mathrm{p}=0.032$ ) and on HOS sport (13.8 units; $95 \% \mathrm{Cl} 0.3$ to $27.3, \mathrm{p}=0.046$ ) (table 4). Of the secondary outcomes, significantly greater improvements in the PT group were observed for HAGOS subscales of symptoms, sport/recreation and quality-of-life (table 4). No betweengroup differences were found for physical activity/sport levels measured by the modified Tegner or the HSAS. Significantly more participants in the PT group $(12 / 14,86 \%)$ reported overall improvement at week 14 compared with controls (6/14, 43\%) (relative risk 2.0; $95 \% \mathrm{Cl} 1.05$ to $3.80, \mathrm{p}=0.034$ )

FAI, femoroacetabular impingement; HAGOS, Copenhagen Hip and Groin Outcome Score; HOS, Hip Outcome Scale; HSAS, Heidelberg Sports Activity Score; iHOT-33, International Hip Outcome Tool; PT, physiotherapy.

24 weeks, the results were inconclusive given the small sample size. The findings provide preliminary evidence supporting the value of a PT rehabilitation programme in speeding progress of postoperative recovery.

Our study was designed with attention to key methodological features to minimise bias including randomisation, concealed allocation and intention-totreat analysis. The primary outcomes were reliable, and valid patient-reported measures were suitable for young active individuals with FAI syndrome. ${ }^{16}$ The delivery of the PT intervention by multiple physiotherapists enhances the generalisability of the findings. Furthermore, as the protocol involved a relatively small number of PT visits $(n=7)$ and emphasised a home rehabilitation programme, it would be reasonably inexpensive to implement.

The study has several limitations. The sample size was small and did not reach our intended target due to difficulty recruiting participants. As this study was conducted in private practice, the surgeons were responsible for identifying potentially eligible patients during their consultation and directing them to the research assistant for further discussion about the study. As this relied on busy surgeons, a number of potentially eligible patients may have been missed. In future studies, it is recommended that the responsibility for participant identification rests with the researchers rather than the surgeons as far as possible. We also modified our eligibility criteria part way through the study to remove the upper age limit of 35 years and include those with bilateral surgery, which bolstered recruitment. Thus, future studies should consider the effect that eligibility criteria have on recruitment. Our recruitment rate of $28 \%$ of those screened was in fact reasonable, but a larger number 
Table 3 Mean (SD) scores on continuous outcome measures across time according to group

\begin{tabular}{lllllll}
\hline & \multicolumn{5}{l}{ Groups } \\
\cline { 2 - 7 } $\begin{array}{l}\text { Primary } \\
\text { outcomes }\end{array}$ & $\begin{array}{l}\text { Baseline } \\
\text { Physiotherapy } \\
(\mathrm{n}=14)\end{array}$ & $\begin{array}{l}\text { Control } \\
(\mathrm{n}=16)\end{array}$ & $\begin{array}{l}\text { Physiotherapy } \\
(\mathrm{n}=14)\end{array}$ & $\begin{array}{l}\text { Control } \\
(\mathrm{n}=14)\end{array}$ & $\begin{array}{l}\text { Physiotherapy } \\
(\mathrm{n}=11)\end{array}$ & $\begin{array}{l}\text { Control } \\
(\mathrm{n}=11)\end{array}$ \\
\hline iHOT-33 & $40.9(15.7)$ & $42.0(17.5)$ & $78.8(17.8)$ & $66.4(20.5)$ & $84.4(12.1)$ & $78.1(16.4)$ \\
\hline HOS sport & $50.9(17.1)$ & $52.1(16.7)$ & $83.6(18.1)$ & $70.8(18.6)$ & $85.0(17.8)$ & $86.0(12.4)$ \\
\hline Secondary outcomes & & & & & \\
\hline HOS ADL & $71.7(11.0)$ & $69.7(13.5)$ & $90.5(9.2)$ & $85.8(10.2)$ & $92.0(10.0)$ & $92.9(6.7)$ \\
\hline HAGOS symptoms & $48.2(15.6)$ & $49.3(16.7)$ & $78.3(15.3)$ & $65.8(15.2)$ & $79.9(10.4)$ & $74.0(16.5)$ \\
\hline HAGOS pain & $68.8(14.9)$ & $61.4(13.4)$ & $87.9(9.7)$ & $81.8(11.2)$ & $88.6(11.1)$ & $88.4(10.6)$ \\
\hline HAGOS ADL & $72.1(13.5)$ & $68.1(14.4)$ & $88.6(10.1)$ & $86.8(10.7)$ & $94.5(7.2)$ & $91.8(9.0)$ \\
\hline HAGOS sport/rec & $35.9(16.9)$ & $43.9(19.3)$ & $77.0(17.8)$ & $61.6(19.8)$ & $81.5(23.4)$ & $78.4(18.6)$ \\
\hline HAGOS participation & $19.6(23.4)$ & $26.6(25.4)$ & $55.4(33.1)$ & $48.2(24.9)$ & $76.1(34.2)$ & $76.1(23.4)$ \\
\hline HAGOS QOL & $29.3(18.0)$ & $37.2(15.2)$ & $66.1(28.8)$ & $53.6(17.6)$ & $70.5(28.2)$ & $68.2(21.7)$ \\
\hline Modified Tegner & $3.9(1.8)$ & $4.3(2.2)$ & $4.8(1.3)$ & $5.1(2.0)$ & $5.5(1.6)$ & $5.6(1.6)$ \\
\hline HSAS & $31.0(18.0)$ & $31.9(21.6)$ & $39.5(14.2)$ & $30.4(20.8)$ & $31.0(8.5)$ & $34.3(17.5)$ \\
\hline
\end{tabular}

iHOT-33, International Hip Outcome Tool (0-100); HOS, Hip Outcome Score (0-100 for subscales sport and activity of daily living); HAGOS, Copenhagen Hip and Groin Outcome Score (0-100 for subscales: pain, symptoms, physical function in daily living, physical function in sport and recreation, participation in physical activities, hip and/or groin-related quality of life); Modified Tegner, Modified Tegner Activity Scale (0-10; $0=$ no participation due to disability, 1-3 activities of daily living/light work, 4-7 physical fitness/moderate-strenuous work, 8-10 competitive sport); HSAS, Heidelberg Sports Activity Score (0-220 with higher scores indicating greater sport activity levels).

$A D L$, activities of daily living.

of surgeons and/or a longer time frame would have been needed to reach our target. These were constrained by funding and timelines. Over $50 \%$ of potentially eligible individuals were unwilling to refrain from postoperative PT rehabilitation and were excluded. Inclusion of a control group whereby the participants consulted a physiotherapist for education and advice rather than a control group with no contact with a physiotherapist may have facilitated participation by patients who were unwilling to refrain from seeing a physiotherapist after surgery. While the dropout rate at the primary time point at 14 weeks was acceptable at $7 \%$, the dropout rate by week 24 was much larger at $27 \%$. The reasons for the dropout are unclear as these participants did not respond to numerous efforts to contact them via various means (eg, telephone, SMS and email). A small financial incentive for completion of questionnaires was provided at the 14-week time point. A similar incentive at the 24-week time point may have improved overall retention rates. Nevertheless, with our small sample, we were still able to demonstrate statistically and clinically significant differences in primary outcomes between groups at our primary time point of 14 weeks with the smaller sample than planned because the effect size of PT was larger than we assumed in our initial sample size calculation. By necessity, patients and physiotherapists were unblinded. We did not include objective measures of physical performance or impairments, so we cannot determine the effect of the intervention on these. Our results cannot be generalised to different rehabilitation programmes with more intensive PT contact and slower progression or to high-level competitive athletes whose functional requirements are greater. We excluded patients who planned to consult a physiotherapist for their rehabilitation due to their high risk of crossover and potential negative expectations about outcomes if they were allocated to the control group. It is possible that this subgroup may respond differently to the PT programme than those who were willing to participate and had some degree of equipoise about PT rehabilitation. Similarly, given the small sample size, we did not formally investigate whether other factors such as age and bilateral surgery moderate outcomes.

Two recent systematic reviews ${ }^{10}$ have highlighted the absence of RCTs investigating the efficacy of adding rehabilitation to hip arthroscopic management of FAI syndrome and as such we cannot directly compare our results to other RCTs. Instead studies have been either observational case series or case reports. Grzybowki et $_{a l^{13}}$ identified 18 such studies and found considerable heterogeneity in rehabilitation programmes as well as poor reporting of specific programme parameters. Differences are apparent in timing of functional progression, often dictated by surgeon-imposed postoperative restrictions related to weight-bearing and hip range of motion, use of therapeutic techniques such as continuous passive motion machines, bracing and types of exercises. Consistent with several other programmes, ours emphasised strategies to improve hip range and muscle strength. ${ }^{1013}$ We also aimed to retrain and strengthen the deep hip rotator muscles, which have a short lever arm and have been suggested to provide fine control of hip joint stability, acting as the 'rotator cuff' of the hip joint. ${ }^{27}{ }^{28}$ Comparisons of our 


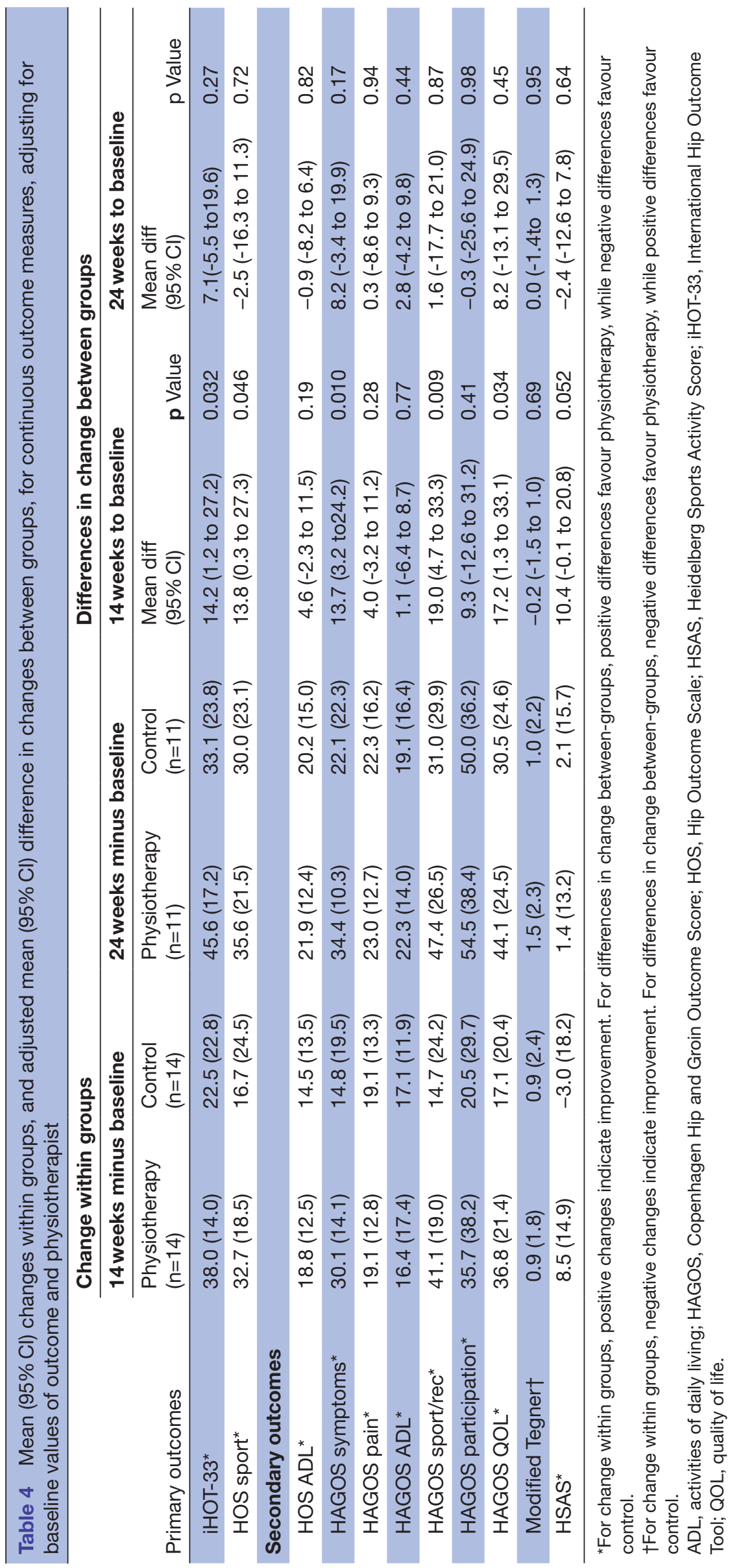


results with those of observational studies are somewhat constrained by differences in terms of outcome measures, follow-up time points post-FAI surgery and patient demographics, particularly age and level of sporting activity. Many studies included older patients to those in our sample and follow-up time points were often longer than 24 weeks, although one study found maximal improvement at this time with no further improvement at 2 years. ${ }^{29}$ Nonetheless, the levels of impairment seen at baseline in our sample and the magnitude of improvement seen in our treatment group are relatively consistent with those reported in observational studies. ${ }^{15}{ }^{29-33}$ For example, we found a 93\% improvement in the iHOT-33 at week 14 and a $110 \%$ improvement at week 24 that is similar to the approximate $90 \%$ and $82 \%$ improvement at 3 months and the $107 \%$ and $97 \%$ at 6 months in men and women, respectively, in the study by Joseph et al. ${ }^{29}$ However, no conclusions can be drawn about the superiority of one rehabilitation protocol over another at this stage.

The mechanisms that led to the greater improvements with the PT-prescribed rehabilitation programme are speculative. First, although not measured, the improvements may be due in part to improved hip rangeof-motion and muscle strength (aims of the rehabilitation programme), as impairments in these have been reported in people with FAI syndrome $e^{34} 35$ and have been related to function. ${ }^{34}$ Second, it is possible that the education, advice and support provided by the physiotherapist and the structured progressive programme gave patients confidence to better manage their recovery. ${ }^{36}$ Third, patients may have been more adherent to performing rehabilitation exercises and activities given the regular contact with the physiotherapist. Last, as self-reported outcomes were used and patients and physiotherapists were not blind to group allocation, it is possible that initial treatment benefits were related, at least partly, to participant expectations and placebo effects. ${ }^{37}$

There was no significant difference between groups at 24 weeks postsurgery. It is possible that benefits of an initial PT programme become less apparent once contact with the physiotherapist ceases. However, the sample size was small and further reduced by the 24-week time point, so that statistical power was limited and sufficient only to detect large treatment effects. Furthermore, three controls crossed-over and sought PT rehabilitation and another two consulted a health professional but were included in the analysis as per intention-to-treat. This may have attenuated differences between groups. Thus, there is potential for a type II error, particularly as the mean between-group difference for change in the iHOT-33 favoured the PT group and exceeded the MCID. Therefore, while it is possible that PT rehabilitation may be beneficial over the longer term, this requires further confirmation.

Although greater improvements in the PT group were noted in a number of functional outcomes, these did not translate into greater increases in levels of sporting and occupational activity, as measured by the modified Tegner and the HSAS. However, this might reflect a limitation of these measures as both are relatively crude and likely not sensitive enough to detect small changes. Return to previous sporting level was not specifically assessed due to difficulties in reliably and accurately measuring this in a heterogeneous cohort such as ours.

As our study used patient-reported outcome measures whose clinimetric properties have been demonstrated in this patient population, our results also provide a baseline against which other protocols can be measured. Such comparisons could point to possible improvements in rehabilitation strategies that can then be introduced and rigorously tested.

\section{CONCLUSION}

Our results suggest that this particular PT rehabilitation programme, as part of the overall arthroscopic management of FAI syndrome, might improve patient-reported outcomes compared with self-directed rehabilitation at 14 weeks postsurgery but not at 24 weeks. However, given the small sample size due to early termination of the study, the results should be considered preliminary and require replication in a larger study before they can inform clinical practice.

\section{Author affiliations}

${ }^{1}$ Department of Physiotherapy, The University of Melbourne, Centre for Health, Exercise and Sports Medicine, School of Health Sciences, Melbourne, Victoria, Australia

${ }^{2}$ Ivanhoe Sports and Physiotherapy Clinic, Melbourne, Victoria, Australia ${ }^{3}$ St Vincent's Private Hospital, East Melbourne, Victoria, Australia

${ }^{4}$ Department of Epidemiology and Preventive Medicine, Monash University, Melbourne, Victoria, Australia

${ }^{5}$ Department of Rheumatology, Royal North Shore Hospital, Sydney, New South Wales, Australia

${ }^{6}$ Institute of Bone and Joint Research, Kolling Institute, University of Sydney, Sydney, New South Wales, Australia

Correction notice This paper has been amended since it was published Online First. Owing to a scripting error, some of the publisher names in the references were replaced with 'BMJ Publishing Group'. This only affected the full text version, not the PDF. We have since corrected these errors and the correct publishers have been inserted into the references.

Acknowledgements Dr Margaret Staples performed the sample size calculation and prepared the randomisation schedule. The YMCA provided the participants in the PT group with free gym and pool access during their 3-month postsurgery treatment period. The orthopaedic surgeons who recruited research participants were Mr Parminder Singh and Mr Andrew Chia. Mr Phong Tran and Mr Cam Fary also identified potential participants but these did not pass screening. The study physiotherapists who provided the PT treatments were: Tony Beecroft, Moonee Ponds/Blackburn; Cara Peake, Ivanhoe; Craig Mansfield, Bendigo; Christian Becker, Narre Warren; Simon Ata, Ivanhoe; and Michael Rafla, Blackburn/Richmond.

Contributors AT, JOD, KLB and RSH conceived the project; KLB and JOD procured the project funding; KLB and RSH coordinated the trial. KLB, RSH, AT, JOD, LS and DJH assisted with protocol design. AT and LS designed the physiotherapy protocol and, along with $\mathrm{KLB}$, trained the physiotherapists. LS recruited the participants and managed the project. KLB wrote the first draft of this manuscript. JK performed the statistical analyses. All authors participated in the trial design, provided feedback on drafts of this paper and read and approved the final manuscript.

Funding This trial was funded by the National Health and Medical Research Council (program grant \#631717), St Vincent's Private Hospital Fitzroy Campus and the Australian Hip Arthroscopy Education and Research Foundation. Kim Bennell (\#1058440) and David Hunter (\#1079777) are supported by NHMRC Fellowships, 
and Rana Hinman is supported by an Australian Research Council Future Fellowship (FT130100175).

Competing interests AT and JOD developed the physiotherapy protocol that was tested and used this in their private clinical practices. JOD is the founder of the Australian Hip Arthroscopy Education and Research Foundation that provided financial support of the project. None of the other authors have any competing interests.

Ethics approval University of Melbourne Human Research Ethics Committee (HREC \#1238190).

Provenance and peer review Not commissioned; externally peer reviewed.

Data sharing statement Statistical code: Available from Dr Kasza (email: jessica. kasza@monash.edu). Data set: available from Dr Bennell (email: k.bennell@ unimelb.edu.au).

Open Access This is an Open Access article distributed in accordance with the Creative Commons Attribution Non Commercial (CC BY-NC 4.0) license, which permits others to distribute, remix, adapt, build upon this work non-commercially, and license their derivative works on different terms, provided the original work is properly cited and the use is non-commercial. See: http://creativecommons.org/ licenses/by-nc/4.0/

(c) Article author(s) (or their employer(s) unless otherwise stated in the text of the article) 2017. All rights reserved. No commercial use is permitted unless otherwise expressly granted.

\section{REFERENCES}

1. Mascarenhas VV, Rego P, Dantas P, et al. Imaging prevalence of femoroacetabular impingement in symptomatic patients, Athletes, and asymptomatic individuals: a systematic review. Eur $J$ Radiol 2016;85:73-95.

2. Anderson SE, Siebenrock KA, Tannast M. Femoroacetabular impingement. Eur J Radiol 2012;81:3740-4.

3. Brunner A, Horisberger M, Herzog RF. Sports and recreation activity of patients with femoroacetabular impingement before and after arthroscopic osteoplasty. Am J Sports Med 2009;37:917-22.

4. Agricola R, Heijboer MP, Bierma-Zeinstra SM, et al. Cam impingement causes osteoarthritis of the hip: a nationwide prospective cohort study (CHECK). Ann Rheum Dis 2013;72:918-23.

5. Kowalczuk M, Yeung M, Simunovic N, et al. Does Femoroacetabular Impingement Contribute to the development of hip osteoarthritis? A systematic review. Sports Med Arthrosc 2015;23:174-9.

6. Griffin DR, Dickenson EJ, O'Donnell J, et al. The Warwick agreement on femoroacetabular impingement syndrome (FAI syndrome): an international consensus statement. Br J Sports Med 2016;50:1169-76.

7. Millennium Research Group. Hip arthroscopy procedures to soar. 2009 http://www.bio-medicine.org/medicine-news-1/HipArthroscopy-Procedures-to-Soar-Through-2013-51045-1/26102009.

8. Sansone M, Ahldén M, Jonasson P, et al. Good results after hip Arthroscopy for femoroacetabular impingement in Top-Level Athletes. Orthop J Sports Med 2015;3:232596711556969.

9. Cooper AP, Basheer SZ, Maheshwari R, et al. Outcomes of hip arthroscopy. A prospective analysis and comparison between patients under 25 and over 25 years of age. Br J Sports Med 2013;47:234-8.

10. Cheatham SW, Enseki KR, Kolber MJ. Postoperative Rehabilitation after hip arthroscopy: a search for the evidence. J Sport Rehabil 2015;24:413-8.

11. Wahoff M, Dischiavi S, Hodge J, et al. Rehabilitation after labral repair and femoroacetabular decompression: criteria-based progression through the return to sport phase. Int J Sports Phys Ther 2014;9:813-26.

12. Bennell KL, O'Donnell JM, Takla A, et al. Efficacy of a physiotherapy rehabilitation program for individuals undergoing arthroscopic management of femoroacetabular impingement - the FAIR trial: a randomised controlled trial protocol. BMC Musculoskelet Disord 2014;15:58.

13. Grzybowski JS, Malloy P, Stegemann C, et al. Rehabilitation following hip arthroscopy - A systematic review. Front Surg 2015;2:21.
14. Tönnis D. Congenital dysplasia and dislocation of the hip in children and adults. Berlin, Germany: Springer-Verlag, 1987.

15. Mohtadi NG, Griffin DR, Pedersen ME, et al. Multicenter Arthroscopy of the Hip Outcomes Research Network. The Development and validation of a self-administered quality-of-life outcome measure for young, active patients with symptomatic hip disease: the International Hip Outcome Tool (iHOT-33). Arthroscopy 2012;28(5):595-610.

16. Hinman RS, Dobson F, Takla A, et al. Which is the most useful patient-reported outcome in femoroacetabular impingement? Testretest reliability of six questionnaires. Br J Sports Med 2014;48.

17. Martin RL. Hip arthroscopy and Outcome Assessment. Oper Tech Orthop 2005;15:290-6.

18. Martin RL, Kelly BT, Philippon MJ. Evidence of validity for the hip outcome score. Arthroscopy 2006;22:1304-11.

19. Martin RL, Philippon MJ. Evidence of validity for the hip outcome score in hip arthroscopy. Arthroscopy 2007;23:822-6.

20. Martin RL, Philippon MJ. Evidence of reliability and responsiveness for the hip outcome score. Arthroscopy 2008;24:676-82.

21. Thorborg K, Hölmich P, Christensen R, et al. The Copenhagen hip and groin outcome score (HAGOS): development and validation according to the COSMIN checklist. Br J Sports Med 2011;45:478-91.

22. Jaeschke R, Singer J, Guyatt GH. Measurement of health status. ascertaining the minimal clinically important difference. Control Clin Trials 1989;10:407-15.

23. Tegner $Y$, Lysholm J. Rating systems in the evaluation of knee ligament injuries. Clin Orthop Relat Res 1985;198:42???49-9.

24. Seeger J, Weinmann S, Schmitt H, et al. The heidelberg sports activity score - a new instrument to evaluate sports activity. Open Orthop J 2013;7:25-32.

25. Frison L, Pocock SJ. Repeated measures in clinical trials: analysis using mean summary statistics and its implications for design. Stat Med 1992;11:1685-704.

26. Papalia R, Del Buono A, Franceschi F, et al. Femoroacetabular impingement syndrome management: arthroscopy or open surgery? Int Orthop 2012;36:903-14.

27. Retchford TH, Crossley KM, Grimaldi A, et al. Can local muscles augment stability in the hip? a narrative literature review. $J$ Musculoskelet Neuronal Interact 2013;13:1-12.

28. Torry MR, Schenker ML, Martin HD, et al. Neuromuscular hip biomechanics and pathology in the athlete. Clin Sports Med 2006;25(2):179-97. vii. doi.

29. Joseph R, Pan X, Cenkus K, et al. Sex differences in SelfReported hip function up to 2 years after arthroscopic surgery for femoroacetabular impingement. Am J Sports Med 2016;44:54-9.

30. Degen RM, Fields KG, Wentzel CS, et al. Return-to-play rates following arthroscopic treatment of femoroacetabular impingement in competitive baseball players. Phys Sportsmed 2016;44:385-90.

31. Tijssen $M$, van Cingel $R$, de Visser $E$, et al. A clinical observational study on patient-reported outcomes, hip functional performance and return to sports activities in hip arthroscopy patients. Phys Ther Sport 2016;20:45-55.

32. Spencer-Gardner L, Eischen JJ, Levy BA, et al. A comprehensive five-phase rehabilitation programme after hip arthroscopy for femoroacetabular impingement. Knee Surg Sports Traumatol Arthrosc 2014;22:848-59.

33. Sansone M, Ahldén M, Jonasson P, et al. Good results after hip arthroscopy for femoroacetabular impingement in Top-Level Athletes. Orthop J Sports Med 2015;3:232596711556969.

34. Nepple JJ, Goljan P, Briggs KK, et al. Hip strength deficits in patients with symptomatic femoroacetabular impingement and labral tears. Arthroscopy 2015;31:2106-11.

35. Diamond LE, Dobson FL, Bennell KL, et al. Physical impairments and activity limitations in people with femoroacetabular impingement: a systematic review. Br J Sports Med 2015;49:230-42.

36. Casartelli NC, Leunig M, Maffiuletti NA, et al. Return to sport after hip surgery for femoroacetabular impingement: a systematic review. $\mathrm{Br} J$ Sports Med 2015;49:819-24.

37. Krogsbøll LT, Hróbjartsson A, Gøtzsche PC. Spontaneous improvement in randomised clinical trials: meta-analysis of three-armed trials comparing no treatment, placebo and active intervention. BMC Med Res Methodol 2009;9:1. 